\title{
Mental Health Professional Consultations and the Prevalence of Mood and Anxiety Disorders Among Immigrants: Multilevel Analysis of the Canadian Community Health Survey
}

Chinenye Nmanma Nwoke ${ }^{1}$, MPH; Udoka Okpalauwaekwe², MBBS, MPH; Hauwa Bwala ${ }^{3}$, DVM, MPH

\footnotetext{
${ }^{1}$ Faculty of Health Sciences, University of Lethbridge, Lethbridge, AB, Canada

${ }^{2}$ Department of Academic Family Medicine, College of Medicine, University of Saskatchewan, Saskatoon, SK, Canada

${ }^{3}$ Western College of Veterinary Medicine, University of Saskatchewan, Saskatoon, SK, Canada
}

\section{Corresponding Author:}

Chinenye Nmanma Nwoke, MPH

Faculty of Health Sciences

University of Lethbridge

4401 University Drive

Lethbridge, AB, T1K 3M4

Canada

Phone: 14035617125

Email: chinenye.nwoke@uleth.ca

\section{Abstract}

Background: There is a significant body of evidence on the link between migration and mental health stressors. However, there has been very little research on the use of mental health services by immigrants in Canada. The prevalence of mental health professional consultations among immigrants, as well as its correlations, are not well understood and remain largely unknown.

Objective: This study aims to examine how specialist mental health visits (to a psychiatrist) differ from general mental health visits (to a family doctor or general practitioner) from immigrants, when compared to visits from those born in Canada, in a nationally representative sample of Canadian adults. This study also examines which group_immigrant or Canadian-born-suffers more from depression or anxiety, 2 of the more common mental health conditions.

Methods: We used data from the Canadian Community Health Survey (CCHS) between the years 2015 and 2016 . The outcome variables included consultation with any mental health professional, consultation with a specialist (psychiatrist), and the prevalence of mood and anxiety disorders. The independent variable was immigrant status. Other variables of interest were adjusted for in the analyses. Multilevel regression models were developed, and all analyses were performed with Stata IC statistical software (version 13.0, StataCorp).

Results: The prevalence of mood and anxiety disorders was significantly lower among immigrants compared with individuals born in Canada; the prevalence of mood disorders was 5.24\% $(389,164 / 7,422,773)$ for immigrants vs. 9.15\% $(2,001,829 / 21,885,625)$ for individuals born in Canada, and the prevalence of anxiety disorders was 4.47\% (330,937/7,410,437) for immigrants vs. 9.51\% $(2,083,155 / 21,898,839)$ for individuals born in Canada. It is expected that individuals with a lower prevalence of mood or anxiety disorders would use mental health services less frequently. However, results show that immigrants, while less likely to consult with any mental health professional $(\mathrm{OR}=0.80,95 \% \mathrm{CI} 0.72-0.88, P<.001)$, were more likely to consult with a psychiatrist $(\mathrm{OR}=1.24,95 \%$ CI 1.04-1.48, $P=.02)$ for their mental health visits when compared to individuals born in Canada.

Conclusions: The results of this study reveal an unusual discord between the likelihood of mental health professional consultations with any mental health professional and mental health visits with psychiatrists among immigrants compared to nonimmigrants in Canada. Mental health initiatives need to be cognizant of the differences in the associated characteristics of consultations for immigrants to better tailor mental health services to be responsive to the unique needs of immigrant populations in Canada.

(JMIR Ment Health 2020;7(9):e19168) doi: 10.2196/19168

\section{KEYWORDS}

immigrants; immigrant mental health; mental health consultations; mood disorders; anxiety disorders; mental health visits; Canadian Community Health Survey 


\section{Introduction}

There is an extensive amount of published literature on mental health conditions in Canada. However, there is a paucity of research on mental health among immigrant populations [1]. A literature review on mental health concerns among Canadian immigrants showed varying trends and patterns. One study noted that immigrants have fewer episodes of mood disorders compared with their native-born counterparts [2]. Contrarily, 2 peer-reviewed articles reported an increased prevalence of mental health conditions such as emotional problems, stress, and increased psychotic disorders among Canadian immigrants $[3,4]$. Enabling factors identified to potentiate mental health problems among immigrants include racial discrimination and immediate difficulties with settling [4,5]. These situations are made worse by the barriers (ie, poverty, experiences of discrimination, stigma, language) faced by immigrants trying to access mental health services [2].

Access to mental health services significantly impacts immigrants, who have been currently identified as one of the most underserved in the Canadian healthcare system [4]. Studies have shown that only $50 \%$ of Canadians with mood disorders and anxiety disorders receive adequate care, in addition to long wait times for counseling and therapy services [6,7]. Specifically, immigrant communities in Canada underutilize mental health services in comparison to national and provincial averages $[6,8]$. For example, immigrant populations in Toronto utilized mental health services significantly less than Canadian-born participants (6\% for recent immigrants, $7 \%$ for longer-term immigrants, and $10 \%$ for Canadian-born participants) [6]. With the increasing influx of immigrants to Canada, addressing gaps in the provision of and access to mental health services, including mental health professionals, among immigrants presents a rapidly emerging challenge.

An understanding of the relationship between mental health professional consultation and its association with immigrant status is largely unknown. The 2015 pan-Canadian survey on mental health services highlighted that $91 \%$ of survey respondents indicated that increasing access to mental health care professionals was a top priority for government action [9]. Examining this relationship is essential to identify potential gaps that need addressing within the mental health care system. Furthermore, understanding the relationship between mental health professional consultations and immigrants could give rise to strategies that would mitigate mental health-induced burdens associated with immigrant transition and assimilation into Canadian culture. More assuredly, a better understanding of immigrant mental health consultation patterns and mental health outcomes encourages patient-centered care in delivering mental health services to Canada's booming immigrant population. The purpose of this study is two-fold and aims to answer the following research questions: (1) What is the variation of past-year mental health consultations (with general health practitioners and with psychiatrists) among immigrants compared to individuals born in Canada? (2) What is the prevalence of mood and anxiety disorders (2 of the most common mental health conditions) among immigrants compared to individuals born in Canada?

\section{Methods}

\section{Data Source}

We obtained data for the study from the 2015-2016 Canadian Community Health Survey (CCHS) [10]. The CCHS is an ongoing, national, cross-sectional survey that collects information on health status, health care utilization, and health determinants for the Canadian population. CCHS employs a cross-sectional study design to collect information related to health status and covers approximately $98 \%$ of the Canadian population aged 12 years and over. The CCHS data structure consists of individuals nested within health regions nested within provinces. CCHS data are collected using computer-assisted in-person and telephone interviewing. Further details on the methodology utilized by the CCHS and its measures can be found on the Statistics Canada webpage [10]. The CCHS uses a multistage stratified cluster design to sample household dwellings of the Canadian population aged 12 years and older living in private households. Excluded from the sampling frame were individuals living on First Nations reserves, institutions, remote regions, and full-time members of the Canadian Forces.

\section{Independent Variable}

The immigrant sample, categorized as a binary variable (yes or no), is defined by Statistics Canada as "persons residing in Canada who were born outside Canada, excluding temporary foreign workers, Canadian citizens born outside Canada, and those with student or working visas" [11]. Participants in the CCHS were asked if they were born in Canada. Those that answered "no" to this question were considered immigrants. Nonimmigrants in this study refer to persons who were born in Canada.

\section{Outcome Variables}

We assessed and analyzed 4 different outcomes related to self-reported responses to past-year mental health consultations. The questions included the following: (1) In the past 12 months, that is, from (date, one year ago) to yesterday, have you seen or talked to a health professional about your emotional or mental health? (yes or no) (2) Whom did you see or talk to? (Psychiatrist? yes or no) (3) Do you have a mood disorder such as depression, bipolar disorder, mania, or dysthymia? (yes or no) (4) Do you have an anxiety disorder such as a phobia, obsessive-compulsive disorder, or a panic disorder? (yes or no). Respondents were to self-report conditions diagnosed by a health professional; however, they were not asked to elaborate on the type of mood disorder diagnosis or the time when the diagnosis occurred. All missing values for the outcome variables were dropped from the dataset during the statistical analyses.

\section{Other Covariates}

Other covariates of interest adjusted for in the analysis include sex (male, female), age, marital status (married/common-law, not married/never married), sense of belonging (strong, weak), working status (not employed, employed), income adequacy (low income, income adequate), highest level of education (high school graduate or less, some post-secondary or more), owned or rented home, cultural or racial origin (Caucasian, visible minority), physical activity level (active, inactive), current 
smoking status (smoker, nonsmoker), and the number of chronic conditions present (zero, 1 or more).

\section{Statistical Analysis}

Individuals were nested within health regions, which were further nested within provinces. Multistage analyses consisting of both descriptive statistics and multilevel logistic regression models were employed to identify relationships among variables measured at the individual level as well as the amount of variation of the effect at the group level (provinces and health regions). Tests of model fit using Akaike information criterion values were done to identify the level of multilevel logistic regression modeling that best fits the data. The estimated prevalence rates of the 2 mental health consultations associated outcomes were calculated for immigrant populations. Multilevel logistic regression modeling was carried out on each of the outcomes. The covariates outlined were assessed as potential characteristics of "any past-year mental health consultation," "past-year psychiatrist consultation," "self-reported diagnosis of mood disorder," and "self-reported diagnosis of anxiety disorder." The confounding effects of control variables on the study outcome variables were assessed at each stage of the model building process; any variable that caused a change of $20 \%$ or more on the regression coefficient of the primary covariates of interest was considered a confounder and was hence retained in the model. We calculated an intraclass correlation coefficient to measure the amount of variation shared by members of the same geographical area and to help us quantify the heterogeneity between the health regions and provinces.

We applied population sample weights to the effect size estimates to make inferences to the Canadian population. We used bootstrap weights provided by Statistics Canada in the CCHS data file to account for the complex sampling design used to calculate the $95 \%$ confidence intervals or the prevalence estimates. Bootstrapping is a technique used to estimate the variance of a statistic. The level of significance was set at 5\%, with two-way analyses in reporting odds ratios and 95\% confidence intervals as well. All analyses were performed using Stata IC (version 13.0, StataCorp) [12].

\section{Results}

\section{Descriptive Statistics}

In the CCHS 2015-2016, 25.33\% (weighted percentage; $7,438,691 / 29,372,184)$ of the Canadian population surveyed $(\mathrm{N}=29,372,184)$ identified themselves as immigrants, with $25.30 \%(1,659,753 / 6,561,120)$ being recent immigrants and $74.70 \%(4,901,367 / 6,561,120)$ being nonrecent immigrants. The results of the descriptive analysis in the CCHS 2015-2016 are displayed in Table 1 . The results show that $8.78 \%$ $(624,617 / 7,110,919)$ of immigrants consulted any mental health professional in the past 12 months, $20.00 \%(124,559 / 622,661)$ had consulted with a psychiatrist, 5.24\% $(389,164 / 7,422,773)$ had mood disorders, and 4.47\% (330,937/7,410,437) had anxiety disorders. The immigrant sample was $49.07 \%$ $(3,649,845 / 7,438,691)$ male and $50.93 \%(3,788,846 / 7,438,691)$ female. The majority of the immigrants were $60+$ years old $(2,017,299 / 4,662,122,43.27 \%)$, married $(4,807,589 / 7,405,342$, $64.92 \%)$, had a strong sense of belonging in the local community $(4,860,802 / 6,988,638, \quad 69.55 \%)$, were employed $(4,451,118 / 6,718,851,66.25 \%)$, earned an adequate income $(5,571,604 / 7,434,459,74.94 \%)$, had some post-secondary education or more $(4,932,147 / 7,306,626,67.50 \%)$, owned their home $(4,991,492 / 7,395,539,67.49 \%)$, were a visible minority $(4,732,248 / 7,340,648,64.47 \%)$, were physically active $(5,345,682 / 7,016,883, \quad 76.18 \%)$, were nonsmokers $(6,565,496 / 7,430,428,88.36 \%)$, and had no chronic conditions $(5,283,483 / 7,387,161,71.52 \%)$. Sensitivity analyses showed that there were no significant differences between outcome responses retained for analysis and those excluded by listwise deletion. 
Table 1. Summary statistics for mental health professional consultation, mental health outcomes, sociodemographic, socioeconomic status, and behavioral variables by immigrant status $(\mathrm{N}=29,372,184)$.

\begin{tabular}{llll}
\hline Outcome variables, $\mathrm{n}(\%)^{\mathrm{a}}$ & Canadian born, & All immigrants, & $P$ values \\
& $\mathrm{n}=21,933,493(74.67 \%)$ & $\mathrm{n}=7,438,691(25.33 \%)$ & \\
\hline
\end{tabular}

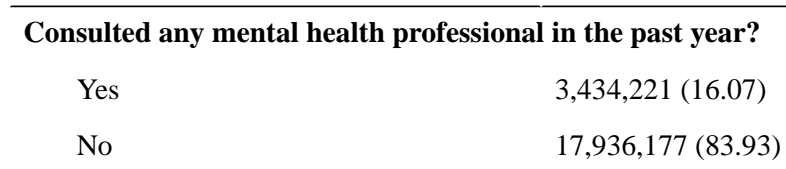

Consulted a psychiatrist in the past year?

$\begin{array}{ll}\text { Yes } & 573,903(16.77) \\ \text { No } & 2,847,760(83.23)\end{array}$

Prevalence of self-reported mood disorders?
Yes
$2,001,829(9.15)$
No
$19,883,796(90.85)$

$<.001$

$624,617(8.78)$

$6,486,302(91.22)$

124,559 (20.00)

$498,102(80.00)$

$389,164(5.24)$

7,033,609 (94.76)

Prevalence of self-reported anxiety disorders?
Yes
$2,083,155(9.51)$
No
$19,815,684(90.49)$

330,937 (4.47)

$7,079,500(95.53)$

Sex
Male
$10,849,193$ (49.46)
Female
$11,086,248(50.54)$

$3,649,845(49.07)$

0.73

$3,788,846(50.93)$

Age category in years

$\begin{array}{ll}<20 & 2,609,211(16.98) \\ 20-39 & 3,407,653(22.18) \\ 40-59 & 3,802,799(24.75) \\ 60+ & 5,545,249(36.09)\end{array}$

\section{Marital status}
Married/common-law
$12,089,338(55.22)$
Not/never married
$9,805,033$ (44.78)

Sense of belonging in the local community
Strong
$14,398,157$ (68.09)
Weak
$6,748,070(31.91)$

Working status last week
Employed
$13,217,420(67.66)$
Not employed
$6,318,019(32.34)$

Income adequacy

Low income household

Income adequate

4,454,184 (20.32)

17,465,194 (79.68)

Highest level of education

High school grad or less

Some post-secondary/more

$9,285,274$ (42.86)

$12,376,542$ (57.14)

Home rented or owned
Owner
$16,559,806(75.81)$
Rented
$5,283,586$ (24.19)

Cultural or racial origin

Caucasian

19,164,163 (92.69)

(9)

422,982 (9.07)

$1,007,521$ (21.61)

$1,214,320(26.05)$

$2,017,299$ (43.27)

$<.001$

4,807,589 (64.92)

$2,597,753$ (35.08)

.65

4,860,802 (69.55)

$2,127,836(30.45)$

.67

$4,451,118$ (66.25)

$2,267,733(33.75)$

$<.001$

$1,862,855$ (25.06)

$5,571,604$ (74.94)

$2,374,479$ (32.50)

4,932,147 (67.50)

4,991,492 (67.49)

2,404,047 (32.51) 


\begin{tabular}{|c|c|c|c|}
\hline Outcome variables, $\mathrm{n}(\%)^{\mathrm{a}}$ & $\begin{array}{l}\text { Canadian born, } \\
\mathrm{n}=21,933,493(74.67 \%)\end{array}$ & $\begin{array}{l}\text { All immigrants, } \\
\mathrm{n}=7,438,691(25.33 \%)\end{array}$ & $P$ values \\
\hline Visible minority & $1,510,714(7.31)$ & $4,732,248(64.47)$ & \\
\hline Physical activity level & & & $<.001$ \\
\hline Active & $16,216,008(82.30)$ & $5,345,682(76.18)$ & \\
\hline Inactive & $3,486,779(17.70)$ & $1,671,201(23.82)$ & \\
\hline Current smoking status & & & $<.001$ \\
\hline Yes & 4,207,071 (19.19) & $864,932(11.64)$ & \\
\hline No & $17,717,523(80.81)$ & $6,565,496(88.36)$ & \\
\hline Chronic conditions & & & .004 \\
\hline No conditions & $15,363,198(70.53)$ & $5,283,483(71.52)$ & \\
\hline 1 or more conditions & $6,418,787(29.47)$ & $2,103,678(28.48)$ & \\
\hline
\end{tabular}

${ }^{\mathrm{a}}$ Canadian Community Health Survey (CCHS) frequency weights have been applied to sample prevalence rate calculations.

From the stratified analysis shown in Table 2, the 2 immigrant $\quad P=.02$ ), consulted a psychiatrist more frequently (21.77\% vs. groups (recent and nonrecent) were significantly different in $16.64 \% ; P=.51)$, and had a higher prevalence of mood $(5.96 \%$ the distribution of the outcome variables and all covariates vs. $3.43 \% ; P<.001)$ and anxiety disorders $(4.92 \%$ vs. $3.18 \%$; $(P<.001)$. Of note is the fact that nonrecent immigrants consulted $\quad P<.001)$. mental health professionals more frequently $(8.95 \%$ vs. $8.32 \%$;

Table 2. Summary statistics for mental health professional consultation and mental health outcomes by recency of immigration.

\begin{tabular}{llll}
\hline Outcome variables, $\mathrm{n}(\%)$ & $\begin{array}{l}\text { All immigrants, } \\
\mathrm{N}=7,438,691(25.33 \%)\end{array}$ & $\begin{array}{l}\text { Recent immigrants }(0-9 \text { years), } \\
\mathrm{n}=1,659,753(25.30 \%)\end{array}$ & $\begin{array}{l}\text { Nonrecent immigrants }(10+\text { years), } \\
\mathrm{n}=4,901,367(74.70 \%)\end{array}$ \\
\hline $\begin{array}{l}\text { Consulted any mental health pro- } \\
\text { fessional in the past year }\end{array}$ & $624,617(8.78)$ & $135,096(8.32)$ & $416,307(8.95)$ \\
$\begin{array}{l}\text { Consulted a psychiatrist in the past } \\
\text { year }\end{array}$ & $124,559(20.00)$ & $22,396(16.64)$ & $90,359(21.77)$ \\
$\begin{array}{l}\text { Prevalence of self-reported mood } \\
\text { disorders }\end{array}$ & $389,164(5.24)$ & $56,854(3.43)$ & $291,501(5.96)$ \\
$\begin{array}{l}\text { Prevalence of self-reported anxiety } \\
\text { disorders }\end{array}$ & $330,937(4.47)$ & $52,702(3.18)$ & $239,839(4.92)$
\end{tabular}

${ }^{\mathrm{a}} \mathrm{Chi}$-square test for differences between recent (0-9 years) and nonrecent (10+ years) immigrant groups.

\section{Multilevel Logistic Regression Analysis}

Tables 3 and 4 show the multilevel logistic regression results with their respective odds ratios and $95 \%$ confidence intervals, adjusted for the primary predictor and other covariates. 
Table 3. Final multivariate multilevel models A and B for immigrant status and mental health professional consultations and psychiatric consultations among respondents in the Canadian Community Health Survey (CCHS) 2015-2016.

\begin{tabular}{|c|c|c|c|c|c|c|}
\hline \multirow[t]{2}{*}{ Variable } & \multicolumn{3}{|c|}{ Model A outcome: any mental health professional ${ }^{\mathrm{a}}$} & \multicolumn{3}{|c|}{$\begin{array}{l}\text { Model B outcome: specialized mental health profession- } \\
\text { al (psychiatrist) }{ }^{\mathrm{a}}\end{array}$} \\
\hline & OR & $95 \% \mathrm{CI}$ & $P$ value & $\mathrm{OR}^{\mathrm{b}}$ & $95 \% \mathrm{CI}$ & $P$ value \\
\hline \multicolumn{7}{|l|}{ Immigrant status } \\
\hline No & Reference & Reference & - & Reference & Reference & - \\
\hline Yes & 0.80 & $0.72-0.88$ & $<.001$ & 1.24 & $1.04-1.48$ & .02 \\
\hline \multicolumn{7}{|l|}{ Sex } \\
\hline Male & Reference & Reference & - & Reference & Reference & - \\
\hline Female & 2.08 & $1.97-2.20$ & $<.001$ & 0.85 & $0.76-0.96$ & .006 \\
\hline Age in years & & & $<.001$ & & & .001 \\
\hline$<20$ & Reference & Reference & - & Reference & Reference & - \\
\hline $20-39$ & 1.07 & $0.93-1.23$ & .36 & 1.03 & $0.85-1.27$ & .74 \\
\hline $40-59$ & 0.96 & $0.83-1.11$ & .56 & 1.24 & $1.02-1.50$ & .03 \\
\hline $60+$ & 0.45 & $0.39-0.52$ & $<.001$ & 0.94 & $0.77-1.15$ & .56 \\
\hline \multicolumn{7}{|l|}{ Marital status } \\
\hline Married/common-law & Reference & Reference & - & Reference & Reference & - \\
\hline Not/never married & 1.43 & $1.35-1.52$ & $<.001$ & 1.40 & $1.22-1.59$ & $<.001$ \\
\hline \multicolumn{7}{|c|}{ Sense of belonging to the local community } \\
\hline Strong & Reference & Reference & - & Reference & Reference & - \\
\hline Weak & 1.46 & $1.38-1.54$ & $<.001$ & 1.39 & $1.24-1.55$ & $<.001$ \\
\hline \multicolumn{7}{|l|}{ Working status last week } \\
\hline Employed & Reference & Reference & - & Reference & Reference & - \\
\hline Not employed & 1.36 & $1.28-1.45$ & $<.001$ & - & - & - \\
\hline \multicolumn{7}{|l|}{ Income adequacy } \\
\hline Income adequate & Reference & Reference & - & Reference & Reference & - \\
\hline Low income household & 1.19 & $1.12-1.27$ & $<.001$ & 0.79 & $0.70-0.91$ & .001 \\
\hline \multicolumn{7}{|l|}{ Highest level of education } \\
\hline High school grad or less & Reference & Reference & - & Reference & Reference & - \\
\hline Some post-secondary/more & 1.23 & $1.17-1.31$ & $<.001$ & - & - & - \\
\hline \multicolumn{7}{|l|}{ Home rented or owned } \\
\hline Owned & Reference & Reference & - & Reference & Reference & - \\
\hline Rented & 1.26 & $1.18-1.34$ & $<.001$ & 1.21 & $1.06-1.38$ & .004 \\
\hline \multicolumn{7}{|l|}{ Cultural or racial origin } \\
\hline Caucasian & Reference & Reference & - & Reference & Reference & - \\
\hline Visible minority & 0.54 & $0.48-0.61$ & $<.001$ & - & - & - \\
\hline \multicolumn{7}{|l|}{ Physical activity level } \\
\hline Active & Reference & Reference & - & Reference & Reference & - \\
\hline Inactive & 0.92 & $0.86-0.99$ & .03 & - & - & - \\
\hline \multicolumn{7}{|l|}{ Current smoking status } \\
\hline No & Reference & Reference & - & Reference & Reference & - \\
\hline Yes & 1.26 & $1.18-1.35$ & $<.001$ & 1.25 & $1.10-1.42$ & .001 \\
\hline Chronic conditions & & & & & & \\
\hline
\end{tabular}




\begin{tabular}{|c|c|c|c|c|c|c|}
\hline \multirow[t]{2}{*}{ Variable } & \multicolumn{3}{|c|}{ Model A outcome: any mental health professional ${ }^{\mathrm{a}}$} & \multicolumn{3}{|c|}{$\begin{array}{l}\text { Model B outcome: specialized mental health profession- } \\
\text { al (psychiatrist) }{ }^{\mathrm{a}}\end{array}$} \\
\hline & OR & $95 \% \mathrm{CI}$ & $P$ value & $\mathrm{OR}^{\mathrm{b}}$ & $95 \% \mathrm{CI}$ & $P$ value \\
\hline No conditions & Reference & Reference & - & Reference & Reference & - \\
\hline 1 or more conditions & 1.40 & $1.32-1.48$ & $<.001$ & 1.15 & $1.02-1.29$ & .03 \\
\hline
\end{tabular}

antraclass correlation coefficient for Model A is $1.08 \%$ and Model B is $1.91 \%$.

${ }^{\mathrm{b}}$ Adjusted for employment status, highest level of education, physical activity, and chronic conditions. 
Table 4. Final multivariate multilevel models $\mathrm{C}$ and $\mathrm{D}$ for immigrant status and the prevalence of mood disorders and anxiety disorders among respondents in the Canadian Community Health Survey (CCHS) 2015-2016.

\begin{tabular}{|c|c|c|c|c|c|c|}
\hline \multirow[t]{2}{*}{ Variable } & \multicolumn{3}{|c|}{ Model C outcome: mood disorders ${ }^{\mathrm{a}}$} & \multicolumn{3}{|c|}{ Model D outcome: anxiety disorders ${ }^{\mathrm{a}}$} \\
\hline & $\mathrm{OR}^{\mathrm{b}}$ & $95 \% \mathrm{CI}$ & $P$ value & $\mathrm{OR}^{\mathrm{c}}$ & $95 \% \mathrm{CI}$ & $P$ value \\
\hline \multicolumn{7}{|l|}{ Immigrant status } \\
\hline No & Reference & Reference & - & Reference & Reference & - \\
\hline Yes & 0.79 & $0.71-0.89$ & $<.001$ & 0.73 & $0.65-0.83$ & $<.001$ \\
\hline \multicolumn{7}{|l|}{ Sex } \\
\hline Male & Reference & Reference & - & Reference & Reference & - \\
\hline Female & 1.88 & $1.76-1.99$ & $<.001$ & 1.93 & $1.81-2.06$ & $<.001$ \\
\hline Age in years & & & $<.001$ & & & $<.001$ \\
\hline$<20$ & Reference & Reference & - & Reference & Reference & - \\
\hline $20-39$ & 1.21 & $1.05-1.39$ & .009 & 1.04 & $0.92-1.18$ & .48 \\
\hline $40-5$ & 1.59 & $1.39-1.82$ & $<.001$ & 0.79 & $0.70-0.89$ & $<.001$ \\
\hline $60+$ & 0.77 & $0.67-0.88$ & $<.001$ & 0.40 & $0.36-0.45$ & $<.001$ \\
\hline \multicolumn{7}{|l|}{ Marital status } \\
\hline Married/common-law & Reference & Reference & - & Reference & Reference & - \\
\hline Not/never married & 1.39 & $1.29-1.49$ & $<.001$ & 1.19 & $1.11-1.28$ & $<.001$ \\
\hline \multicolumn{7}{|c|}{ Sense of belonging to local community } \\
\hline Strong & Reference & Reference & - & Reference & Reference & - \\
\hline Weak & 1.95 & $1.83-2.08$ & $<.001$ & 1.66 & $1.56-1.77$ & $<.001$ \\
\hline \multicolumn{7}{|l|}{ Working status last week } \\
\hline Employed & Reference & Reference & - & Reference & Reference & - \\
\hline Not employed & 1.61 & $1.50-1.72$ & $<.001$ & 1.60 & $1.49-1.72$ & $<.001$ \\
\hline \multicolumn{7}{|l|}{ Income adequacy } \\
\hline Income adequate & Reference & Reference & - & Reference & Reference & - \\
\hline Low income household & 1.55 & $1.44-1.67$ & $<.001$ & 1.39 & $1.29-1.50$ & $<.001$ \\
\hline \multicolumn{7}{|l|}{ Highest level of education } \\
\hline High school grad or less & Reference & Reference & - & Reference & Reference & - \\
\hline Some post-secondary/more & 1.08 & $1.01-1.15$ & .02 & - & - & - \\
\hline \multicolumn{7}{|l|}{ Home rented or owned } \\
\hline Owned & Reference & Reference & - & Reference & Reference & - \\
\hline Rented & 1.44 & $1.33-1.54$ & $<.001$ & 1.39 & $1.29-1.50$ & $<.001$ \\
\hline \multicolumn{7}{|l|}{ Cultural or racial origin } \\
\hline Caucasian & Reference & Reference & - & Reference & Reference & - \\
\hline Visible minority & 0.51 & $0.44-0.59$ & $<.001$ & 0.63 & $0.54-0.72$ & $<.001$ \\
\hline \multicolumn{7}{|l|}{ Physical activity level } \\
\hline Active & Reference & Reference & - & Reference & Reference & - \\
\hline Inactive & - & - & - & - & - & - \\
\hline \multicolumn{7}{|l|}{ Current smoking status } \\
\hline No & Reference & Reference & - & Reference & Reference & - \\
\hline Yes & 1.67 & $1.55-1.79$ & $<.001$ & 1.74 & $1.62-1.87$ & $<.001$ \\
\hline \multicolumn{7}{|l|}{ Chronic conditions } \\
\hline No conditions & Reference & Reference & - & Reference & Reference & - \\
\hline
\end{tabular}




\begin{tabular}{|c|c|c|c|c|c|c|}
\hline \multirow[t]{2}{*}{ Variable } & \multicolumn{3}{|c|}{ Model C outcome: mood disorders ${ }^{\mathrm{a}}$} & \multicolumn{3}{|c|}{ Model D outcome: anxiety disorders ${ }^{\mathrm{a}}$} \\
\hline & $\mathrm{OR}^{\mathrm{b}}$ & $95 \% \mathrm{CI}$ & $P$ value & $\mathrm{OR}^{\mathrm{c}}$ & $95 \% \mathrm{CI}$ & $P$ value \\
\hline 1 or more conditions & 1.74 & $1.63-1.86$ & $<.001$ & 1.73 & $1.61-1.85$ & $<.001$ \\
\hline
\end{tabular}

${ }^{\mathrm{a}}$ Intraclass correlation coefficient for Model C is $2.31 \%$ and Model D is $1.34 \%$.

${ }^{\mathrm{b}}$ Adjusted for physical activity level.

${ }^{\mathrm{c}}$ Adjusted for the highest level of education and physical activity level.

\section{Consulted Any Mental Health Professional (Model A)}

Immigrants had lesser odds of consulting any mental health professional than nonimmigrants (OR 0.8, 95\% CI 0.7-0.9). Even when predisposing (age groups and sex) and enabling characteristics (owned home, employment status, etc) were taken into account, the association between immigrant status and any mental health professional consultation persisted (Table 3, Model A). The sociodemographic variables showed that the odds of consulting any mental health professional were almost twice as high for females when compared to males (OR 2.1, 95\% CI 2.0-2.2). However, not married and never married respondents had significantly higher odds of consulting any mental health professional than married individuals (OR 1.4, 95\% CI 1.4-1.5). Interestingly, individuals that felt a very weak sense of belonging to their local communities (OR 1.5, 95\% CI 1.4-1.5), individuals between the ages of 20-44 years (OR 1.1, 95\% CI 0.9-1.2), individuals from low-income households (OR 1.2 , 95\% CI 1.1-1.3), individuals that lived in rental accommodations (OR 1.3, 95\% CI 1.2-1.3), smokers (OR 1.3, 95\% CI 1.2-1.4), and individuals with 1 or more chronic conditions (OR 1.4, 95\% CI 1.3-1.5) all had significantly higher odds of consulting any mental health professional in comparison with their respective reference groups.

\section{Consulted a Psychiatrist (Model B)}

Immigrants had higher odds of consulting with a psychiatrist than their nonimmigrant counterparts (OR 1.2, 95\% CI 1.0-1.5). When predisposing (age groups and sex) and enabling characteristics (marital status, owned home, employment status, etc) were taken into account, the association between immigrant status and consultation of a psychiatrist persisted (Table 3, Model B). The sociodemographic variables showed that females had lesser odds of consulting a psychiatrist than males (OR 0.9, 95\% CI 0.8-1.0). In addition, not married and never married individuals had significantly higher odds of consulting with a psychiatrist than their married and common-law counterparts (OR 1.4, 95\% CI 1.2-1.6). Similarly, individuals that felt a very weak sense of belonging to their local communities (OR 1.4, 95\% CI 1.2-1.6), those that lived in rental accommodations (OR 1.2, 95\% CI 1.1-1.4), current smokers (OR 1.3, 95\% CI 1.1-1.4), those with 1 or more chronic conditions (OR 1.2, 95\% CI 1.0-1.3), and those aged 45-64 years (OR 1.2, 95\% CI 1.0-1.5) all had significantly elevated odds of consulting with a psychiatrist compared to their respective reference groups. Individuals from low-income households had lower odds of consulting a psychiatrist (OR $0.8,95 \%$ CI 0.7-0.9).

\section{Presence of Mood or Anxiety Disorder (Model C and Model D)}

In examining 2 of the most common psychiatric disorders, Table 4 shows that the immigrants had significantly lesser odds of having mood disorders (OR $0.8,95 \%$ CI $0.7-0.9$ ) and anxiety disorders (OR 0.7, 95\% CI 0.7-0.8). Females, younger age groups, not married and never-married individuals, individuals with a weak sense of belonging to the local community, unemployed individuals, current smokers, and those with 1 or more chronic conditions also had increased odds of having mood or anxiety disorders. Individuals from visible minority groups had lesser odds of having both a mood disorder (OR 0.5, 95\% CI 0.4-0.6) and an anxiety disorder (OR 0.6, 95\% CI 0.5-0.7). These associations were both significant $(P<.001)$.

Significant results between health region and provincial variation were identified for all outcome variables. After completing all 4 final models, it was found that the proportion of group-level variability explained by the health region level and provincial factors included in the models ranged from $1-2 \%$ for the odds of positively consulting any mental health professional, consulting a psychiatrist, and the presence of either a mood or anxiety disorder. We used 2-tailed Wald tests with statistical significance set at $P=.05$ to determine the statistical significance of the determinants in the final models, and only statistically significant variables were included in the final models shown in Tables 3 and 4. Model diagnostics were assessed using the Akaike information criterion at each stage of the model building process to ensure model fit and to check for outliers. All residuals were shown to be within acceptable levels.

\section{Discussion}

\section{Principal Findings}

The main objective of this study is to examine differences in any mental health professional consultation and psychiatrist consultations among immigrant and nonimmigrant groups in Canada. This study also examines the prevalence of mood and anxiety disorders among immigrants to Canada compared to those born in Canada.

The study results revealed that Canadian-born populations were more likely to suffer from self-reported diagnoses of mood and anxiety disorders compared with immigrant populations in Canada. This finding is consistent with other Canadian studies $[13,14]$ and studies done in the United States [15-17]. Ideally, it would be expected that individuals with a more significant burden of mood or anxiety disorders would use mental health services more often. However, we found that immigrants, when compared to Canadian-born populations, were less likely to consult with any mental health professional (eg, psychologists, 
social workers, general physicians) and were more likely to consult with a psychiatrist for their mental health visits. This suggests an apparent primary care gap in the services received by immigrants and could be an indication of the reported severity of mental health issues immigrants commonly face $[18,19]$. The implication of this gap is a delay in the reception of care, leading to further deterioration of mental health with increasing cost implications to the individual and health care system.

It is common knowledge that psychiatrists typically tend to handle more severe cases of mental health issues than family physicians or other health care providers. Additionally, there is evidence in the literature showing how challenges with language, stigma, and other difficulties prevent immigrants from promptly accessing the mental health care they need [20-23]. These observations, taken into considerations with our findings of increased use of psychiatrists' services, give further credibility to our position of a visible primary care gap, which could be mitigated by promoting support for the mental health of immigrants at an early stage of illness through primary health care services.

An alternative explanation for the frequent use of psychiatrists over other primary care providers could be that immigrants are more aware of the services that psychiatrists provide based on traditional knowledge from their home countries, in comparison to mental health services offered by psychologists, social workers, and family physicians. It is also possible that our findings might have been skewed to the individual preference for specialist care (ie, psychiatrists) [24]; however, a closer look at this study attributed its findings to the local presence of a psychiatric hospital and an extensive network of community-based organizations involved in mental health.

Interestingly, when respondents were stratified by self-reported diagnosis of mood and anxiety disorders, immigrants were more likely to consult a psychiatrist for their mental health than any other mental health professional as compared to Canadian-born populations. These findings-along with the identified effect of a sense of belonging, marital status, living arrangement, and being from a visible minority immigrant group-provide additional support for the need to explore mental health service utilization. The observed risk factors associated with mental health professional consultations in this study overlapped with many of the determinants found in other literature [25-27]. This study found that immigrants from a visible minority population (ie, racialized immigrants) were much less likely to report mental health consultations. The stressors of migration and resettlement have profound mental health implications, including mental health professional consultations. Long wait times, language barriers, lack of culturally safe care, the proximity of mental health services and difficulties with transportation, challenges in finding timely child care, and lack of suitable e-mental health technology implementation have been cited as primary reasons for unmet mental health care needs $[7,21,28]$. More importantly, a number of cultural factors (ie, differences in language and ethnicity of care providers) related to shame, stigma, and preference for help-seeking from family members have been cited as possible reasons for why Chinese populations in Canada persistently report the lowest levels of mental health service utilization [1]. These gaps warrant further research inquiry.

A few studies have reported gender differences in mental health-related consultations to be often nonexistent [29-31]. In our study, we found that female immigrants were more likely to consult with any mental health professional and with a psychiatrist as compared with male immigrants. However, men were more likely to have an inadequate number of mental health consultations compared with women, which could be due to gender differences in accessing health care (ie, women being more likely to have and keep to regular medical appointments with their family physician) [32-34]. This could imply that the mental health of immigrant men may be worse than that of immigrant women; nonetheless, further research is necessary to corroborate this claim further.

Our study provides additional insight into the type of mental health professional consultation an immigrant is more likely to seek. It is also insightful in offering education about alternative sources of mental health care to immigrant populations in Canada. Additionally, this could be useful for policymakers and service providers in targeting mental health funds and programs to vulnerable groups. Ultimately, addressing this issue could lead to increased health-related quality of life, improved productivity in the workforce, and decreased cost of medical resources targeted towards immigrant mental health care. More research is warranted to explore further and to understand better the discordance between mental health professional visits and psychiatric visits in general among immigrant and Canadian-born populations in Canada. Even so, a more comprehensive version of the CCHS is needed, especially one that takes into account immigrant-specific measures of variables such as language barriers in accessing mental health care, mental health stigma, level of mistrust of mental health services, the availability of culturally safe mental health care, and preferred immigrant help-seeking behaviors for mental health. These will help researchers and clinicians to better assess how mental health promotion programs can be tailored to address the specific mental health needs of immigrant populations in Canada.

\section{Study Limitations}

Limitations of this study were, for the most part, related to the use of CCHS data. First, there were a limited number of mental health-specific variables in the dataset. This fact, in addition to the dichotomy of almost all variable responses, made it impossible to explore some other factors (for example, the sex of mental health professionals, and reasons for not consulting any mental health professional). Second, the CCHS data, although almost representative of the Canadian population, does not have data for on-reserve Aboriginal populations. Further research of this highly vulnerable group could have been explored and contrasted to findings among immigrant groups, as both groups face similar barriers in accessing culturally appropriate health and mental health care services in Canada. Third, as with all epidemiological studies employing cross-sectional data, we cannot make inferences about the temporality of events. Fourth, diagnostic tests for all models showed residuals within acceptable levels, hence implying the absence of outliers and increasing the reliability of the model. 
However, caution should be applied due to the limitations in the CCHS publicly available dataset. Lastly, the CCHS has the potential for recall bias as it requires participants to remember past events rather than corroborating with medical records. In addition, the outcome variables of mental health consultation (any and psychiatric) were based on a single survey item.

Despite these highlighted limitations, the use of the CCHS for this analysis provided comparative data from a large Canadian sample, which allowed for inferences to be made about the Canadian population as a whole. These findings could be further explored in future research with adjustments to the survey tools over time, especially as it relates to the inclusion of culturally specific variables.

\section{Conclusions}

This study found that the majority of immigrants who seek mental health care consult the specialist services of a psychiatrist. As psychiatrists are known to deal with more complex cases of mental health, there is a crucial need for open conversations on mental health between family doctors and immigrants, as this is usually their first contact with the Canadian health care system. An expansion of the care role of family doctors may be a possible avenue to pursue to improve mental health service access for immigrant populations. A weak sense of belonging was strongly associated with past-year mental health consultation for immigrant populations. Mental health initiatives and policies need to be embedded in immigrant settlement initiatives to ensure that immigrants are better connected to their local communities. These could help immigrants get the right information about mental health in a timely manner and ultimately improve overall health and wellbeing. Cross-sectoral and holistic approaches are thus needed to move towards the comprehensive provision of timely and culturally safe mental health care delivery. This study examined conventional mental health professional consultation. Further research is required to understand how immigrant populations use other unconventional mental health supports as they relate to help-seeking behaviors for mental health; these can include social support networks, faith-based support, family or friend support, and spiritual or cultural services.

\section{Acknowledgments}

The authors wish to thank Statistics Canada for making available the 2015-2016 Canadian Community Health Survey (CCHS), Public Use Microdata File.

\section{Authors' Contributions}

This study was conceptualized and designed by $\mathrm{CN}$, who acquired the data, analyzed and interpreted the data, and drafted the manuscript. CN had full access to all the data in the study and had final responsibility for the decision to submit for publication. UO and HB contributed to the data interpretation, writing, critical review, and revision of the manuscript. All authors provided intellectual content, reviewed, edited, and amended the manuscript. All authors gave final approval of the version to be published and agreed to be accountable for all aspects of the work.

\section{Conflicts of Interest}

None declared.

\section{References}

1. Fang L. A sociocultural perspective of mental health service use by Chinese immigrants. Settlement of Newcomers to Canada, 12, 152. Toronto, ON: University of Toronto; 2010. URL: https://tspace.library.utoronto.ca/bitstream/1807/94702/ 1/A\%20Sociocultural\%20perspective Fang.pdf [accessed 2020-04-01]

2. Fung K, Guzder J. Canadian Immigrant Mental Health. In: Moussaoui D, Bhugra D, Ventriglio A, editors. Mental Health and Illness in Migration. Mental Health and Illness Worldwide. Singapore: Springer; Apr 18, 2018:1-21.

3. Islam F. Immigrating to Canada During Early Childhood Associated with Increased Risk for Mood Disorders. Community Ment Health J 2015 Aug;51(6):723-732 [FREE Full text] [doi: 10.1007/s10597-015-9851-y] [Medline: 25725782]

4. Robert AM, Gilkinson T. Mental health and well-being of recent immigrants in Canada: Evidence from the Longitudinal Survey of Immigrants to Canada. Citizenship and Immigration Canada. 2012 Nov. URL: https://www.canada.ca/content/ dam/ircc/migration/ircc/english/pdf/research-stats/mental-health.pdf [accessed 2020-04-04]

5. George U, Thomson MS, Chaze F, Guruge S. Immigrant Mental Health, A Public Health Issue: Looking Back and Moving Forward. Int J Environ Res Public Health 2015 Oct 27;12(10):13624-13648 [FREE Full text] [doi: 10.3390/ijerph121013624] [Medline: 26516884]

6. O'Campo P. The mental health and wellbeing of immigrants in Toronto: A report to the ministry of health and long-term care. Toronto: Li Ka Shing Knowledge Institute. Keenan Research Centre. St. Michael's Hospital. Toronto, ON: Centre for Research on Inner City Health; 2012. URL: http://www.ontariohealthprofiles.ca/onmarg/additionalResources/ CRICH RF-v3-i2\%202012.pdf

7. Gadalla TM. Ethnicity and Seeking Treatment for Depression: A Canadian National Study. Canadian Ethnic Studies 2010;41(3):233-245 [FREE Full text] [doi: 10.1353/ces.2010.0042] 
8. Kirmayer LJ, Weinfeld M, Burgos G, du Fort GG, Lasry JC, Young A. Use of health care services for psychological distress by immigrants in an urban multicultural milieu. Can J Psychiatry 2007 May;52(5):295-304 [FREE Full text] [doi: 10.1177/070674370705200504] [Medline: 17542380]

9. 2015 Pan-Canadian Survey Raises Warning Signs on Mental Health Services. Mood Disorders Society of Canada. Belleville, ON; 2015. URL: https://mdsc.ca/research/2015-mental-health-care-system-survey-results/ [accessed 2020-04-01]

10. Canadian Community Health Survey - Annual Component (CCHS).Data sources and methodology. Statistics Canada. Ottawa, ON; 2016 Jan 11. URL: https://www23.statcan.gc.ca/imdb/p2SV.pl?Function=getSurvey\&Id=259374\#a2 [accessed 2020-02-15]

11. Dictionary, Census of Population, 2016. Immigrant status. Statistics Canada. Ottawa, ON; 2017 Oct 25. URL: https:/ /www12.statcan.gc.ca/census-recensement/2016/ref/dict/pop148-eng.cfm [accessed 2020-03-02]

12. StataCorp. Stata Statistical Software: Release 13. College Station, TX: StataCorp LP; 2013. URL: https://www.oalib.com/ references/5814356

13. Fairbrother N, Janssen P, Antony MM, Tucker E, Young AH. Perinatal anxiety disorder prevalence and incidence. J Affect Disord 2016 Aug;200:148-155 [FREE Full text] [doi: 10.1016/j.jad.2015.12.082] [Medline: 27131505]

14. Falah-Hassani K, Shiri R, Vigod S, Dennis CL. Prevalence of postpartum depression among immigrant women: A systematic review and meta-analysis. J Psychiatr Res 2015 Nov;70:67-82 [FREE Full text] [doi: 10.1016/j.jpsychires.2015.08.010] [Medline: 26424425]

15. Alegría M, Álvarez K, DiMarzio K. Immigration and Mental Health. Current Epidemiology Reports 2017 Jun;4(2):145-155 [FREE Full text] [doi: 10.1007/s40471-017-0111-2]

16. Breslau J, Borges G, Hagar Y, Tancredi D, Gilman S. Immigration to the USA and risk for mood and anxiety disorders: variation by origin and age at immigration. Psychological Medicine 2008 Nov 12;39(7):1117-1127 [FREE Full text] [doi: $10.1017 / \mathrm{s} 0033291708004698]$

17. Alegría M, Canino G, Shrout PE, Woo M, Duan N, Vila D, et al. Prevalence of mental illness in immigrant and non-immigrant U.S. Latino groups. American Journal of Psychiatry 2008 Mar;165(3):359-369 [FREE Full text] [doi: 10.1176/appi.ajp.2007.07040704] [Medline: 18245178]

18. Wolf S, Hahn E, Dettling M, Nguyen MH, Wingenfeld K, Stingl M, et al. Migration-Related Stressors and Their Effect on the Severity Level and Symptom Pattern of Depression among Vietnamese in Germany. Depression Research and Treatment 2017 Aug 22:1-9 [FREE Full text] [doi: 10.1155/2017/8930432] [Medline: 28912969]

19. Kirmayer L, Narasiah L, Munoz M, Rashid M, Ryder AG, Guzder J, et al. Common mental health problems in immigrants and refugees: general approach in primary care. CMAJ 2011 Sep 06;183(12):E959-E967 [FREE Full text] [doi: 10.1503/cmaj.090292] [Medline: 20603342]

20. Khanlou N, Haque N, Skinner A, Mantini A, Kurtz Landy C. Scoping Review on Maternal Health among Immigrant and Refugee Women in Canada: Prenatal, Intrapartum, and Postnatal Care. Journal of Pregnancy 2017 Jan 21;2017:8783294 [FREE Full text] [doi: 10.1155/2017/8783294] [Medline: 28210508]

21. Hansson E, Tuck A, McKenzie K. Improving mental health services for immigrant, refugee, ethno-cultural and racialized groups: Issues and options for service improvement. Mental Health Commission of Canada. Ottawa, ON; 2010. URL: https://www.mentalhealthcommission.ca/sites/default/files/Diversity_Issues_Options_Report_ENG_0_1.pdf [accessed 2020-03-17]

22. Pumariega AJ, Rothe E, Pumariega JB. Mental health of immigrants and refugees. Community Ment Health Journal 2005 Oct;41(5):581-597 [FREE Full text] [doi: 10.1007/s10597-005-6363-1] [Medline: 16142540]

23. Ali J. Mental health of Canada's immigrants. Supplement to Health Reports 2002;13(Statistics Canada, Catalogue 82-003):1-11 [FREE Full text]

24. Fleury MJ, Grenier G, Bamvita JM, Caron J. Determinants and patterns of service utilization and recourse to professionals for mental health reasons. BMC Health Serv Res 2014 Apr 08;14:161 [FREE Full text] [doi: 10.1186/1472-6963-14-161] [Medline: 24712834]

25. Diaz-Granados N, Georgiades K, Boyle MH. Regional and individual influences on use of mental health services in Canada. Canadian Journal of Psychiatry 2010 Jan;55(1):9-20 [FREE Full text] [doi: 10.1177/070674371005500103] [Medline: 20113539]

26. Sareen J, Cox BJ, Afifi TO, de Graaf R, Asmundson GJG, ten Have M, et al. Anxiety disorders and risk for suicidal ideation and suicide attempts: a population-based longitudinal study of adults. Arch Gen Psychiatry 2005 Nov;62(11):1249-1257 [FREE Full text] [doi: 10.1001/archpsyc.62.11.1249] [Medline: 16275812]

27. Vasiliadis HM, Lesage A, Adair C, Boyer R. Service use for mental health reasons: cross-provincial differences in rates, determinants, and equity of access. Canadian Journal of Psychiatry 2005 Sep;50(10):614-619 [FREE Full text] [doi: 10.1177/070674370505001007] [Medline: 16276852]

28. Strudwick G, Impey D, Torous J, Krausz RM, Wiljer D. Advancing E-Mental Health in Canada: Report From a Multistakeholder Meeting. JMIR Ment Health 2020 Apr 30;7(4):e19360 [FREE Full text] [doi: 10.2196/19360] [Medline: $\underline{32330114]}$ 
29. Elhai JD, Ford JD. Correlates of mental health service use intensity in the National Comorbidity Survey and National Comorbidity Survey Replication. Psychiatric Services 2007 Aug;58(8):1108-1115. [doi: 10.1176/ps.2007.58.8.1108] [Medline: 17664523 ]

30. Fikretoglu D, Elhai JD, Liu A, Richardson JD, Pedlar DJ. Predictors of likelihood and intensity of past-year mental health service use in an active Canadian military sample. Psychiatr Serv 2009 Mar;60(3):358-366 [FREE Full text] [doi: 10.1176/ps.2009.60.3.358] [Medline: 19252049]

31. Lindsay Nour BM, Elhai JD, Ford JD, Frueh BC. The role of physical health functioning, mental health, and sociodemographic factors in determining the intensity of mental health care use among primary care medical patients. Psychological Services 2009;6(4):243-252 [FREE Full text] [doi: 10.1037/a0017375]

32. Taber JM, Leyva B, Persoskie A. Why do people avoid medical care? A qualitative study using national data. Journal of General Internal Medicine 2015 Mar;30(3):290-297 [FREE Full text] [doi: 10.1007/s11606-014-3089-1] [Medline: 25387439]

33. Cox D. Gender differences in professional consultation for a mental health concern: A Canadian population study. Canadian Psychology/Psychologie canadienne 2014;55(2):68-74 [FREE Full text] [doi: 10.1037/a0036296]

34. Bidmon S, Terlutter R. Gender Differences in Searching for Health Information on the Internet and the Virtual Patient-Physician Relationship in Germany: Exploratory Results on How Men and Women Differ and Why. Journal of Medical Internet Research 2015;17(6):e156 [FREE Full text] [doi: 10.2196/jmir.4127] [Medline: 26099325]

\section{Abbreviations}

CCHS: Canadian Community Health Survey

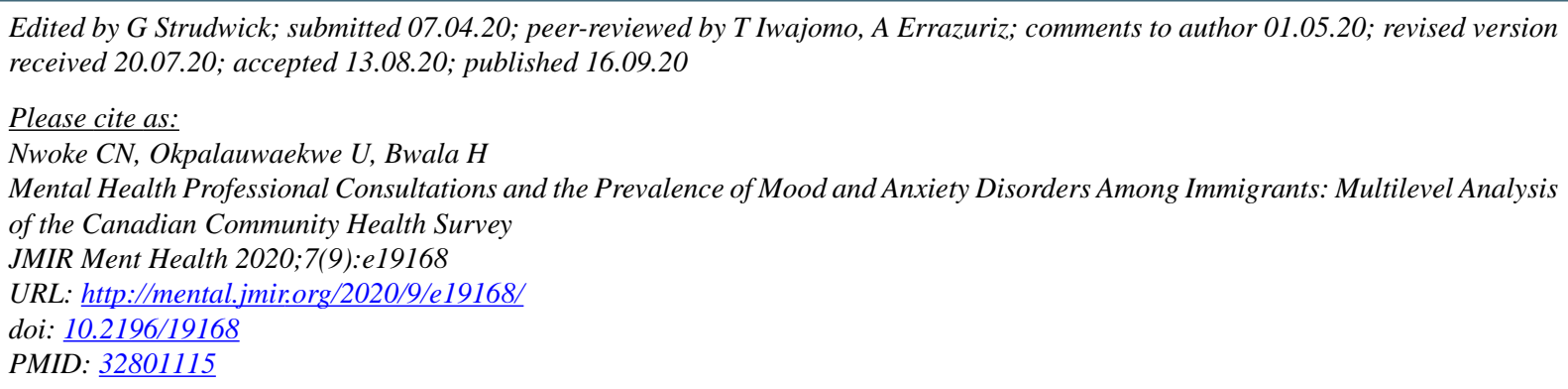

CChinenye Nmanma Nwoke, Udoka Okpalauwaekwe, Hauwa Bwala. Originally published in JMIR Mental Health (http://mental.jmir.org), 16.09.2020. This is an open-access article distributed under the terms of the Creative Commons Attribution License (https://creativecommons.org/licenses/by/4.0/), which permits unrestricted use, distribution, and reproduction in any medium, provided the original work, first published in JMIR Mental Health, is properly cited. The complete bibliographic information, a link to the original publication on http://mental.jmir.org/, as well as this copyright and license information must be included. 